\title{
Palladium-catalysed
}

\section{carboformylation of alkynes using \\ acid chlorides as a dual carbon monoxide and carbon source}

\section{Journal Article}

Author(s):

Lee, Yong Ho (1); Denton, Elliott (1); Morandi, Bill

Publication date:

2021-02

Permanent link:

https://doi.org/10.3929/ethz-b-000467053

Rights / license:

In Copyright - Non-Commercial Use Permitted

Originally published in:

Nature Chemistry 13(2), https://doi.org/10.1038/s41557-020-00621-x

Funding acknowledgement:

757608 - Shuttle Catalysis for Reversible Molecular Construction (EC) 
This document is the Accepted Manuscript version of a Published Work that appeared in final form in Nature Chemistry, copyright $\odot$ Springer Nature after peer review and technical editing by the publisher. To access the final edited and published work see https://doi.org/10.1038/s41557-020-00621-x

\title{
Palladium-catalysed carboformylation of alkynes using acid chlorides as a dual carbon monoxide and carbon source
}

Yong Ho Lee, Elliott H. Denton, Bill Morandi*

ETH Zürich, Vladimir-Prelog-Weg 3, HCI, 8093 Zürich, Switzerland.

*Correspondence to: bill.morandi@org.chem.ethz.ch

\begin{abstract}
Hydroformylation, a reaction which installs both a $\mathrm{C}-\mathrm{H}$ bond and an aldehyde group across an unsaturated substrate, is one of the most important catalytic reactions both in industry and academia. Given the synthetic importance of creating new $\mathrm{C}-\mathrm{C}$ bonds, the development of carboformylation reactions, wherein a new $\mathrm{C}-\mathrm{C}$ bond is formed instead of a $\mathrm{C}-\mathrm{H}$ bond, would bear enormous synthetic potential to rapidly increase molecular complexity in the synthesis of valuable aldehydes. However, the demanding complexity inherent in a fourcomponent reaction, utilizing an exogenous $\mathrm{CO}$ source, has made the development of a direct carboformylation reaction a formidable challenge. Here, we describe a palladium catalysed strategy which uses readily available aroyl chlorides as a carbon electrophile and CO source, in tandem with a sterically congested hydrosilane, to perform a stereoselective carboformylation of alkynes. An extension of this protocol to four chemodivergent carbonylations further highlights the creative opportunity offered by this strategy in carbonylation chemistry.
\end{abstract}

Carbonylation reactions using carbon monoxide $(\mathrm{CO})$ constitute an industrial core technology. They provide a direct and atom-economic strategy to convert, on a multimillion ton-scale per year, bulk chemicals to various carbonyl-containing compounds and their derivatives, which are essential commodity products in daily life $\mathrm{e}^{1-4}$. Due to the importance of these reactions in preparative chemistry, intense academic and industrial research has been dedicated to the development of more environmentally benign and robust catalyst systems as well as highly chemo, regio- and stereoselective carbonylation reactions ${ }^{5-14}$. The seminal discovery of transition-metal 
catalyzed carbonylations was reported by Reppe, which utilizes feedstock alkenes, $\mathrm{CO}$ and a nucleophile to produce the corresponding carbonylated products ${ }^{4}$. Later, significant progress in the field of carbonylation chemistry was made by Heck in 1974, where a palladium catalyst allowed for the carbonylation of carbon electrophiles with various nucleophiles under a pressurized $\mathrm{CO}$ atmosphere to generate the corresponding carbonyl-containing compounds ${ }^{6,11}$. Simultaneously, significant research has focused on vanquishing the hazards associated with the use of toxic and highly flammable $\mathrm{CO}$, as well as avoiding the use of pressurized reactors to facilitate laboratory use. Among these strategies, the use of $\mathrm{CO}$ surrogates and two-chamber systems operating under mild conditions, as well as CO transfer by shuttle catalysis and single bond metathesis strategies, have emerged as valuable alternatives ${ }^{15-22}$.

Among all carbonylation reactions, catalytic hydroformylation, the addition of $\mathrm{CO}$ and $\mathrm{H}_{2}$ across unsaturated substrates, is an essential reaction to access a wide set of functionalized aldehyde products. Both the industrial and academic importance of this reaction are clearly highlighted by the large volumes of aldehydes it generates annually (>12 million tons), as well as the continuous filing and publication of new patents and manuscripts in this area $(>10,000)^{2}$. A key feature of hydroformylation is the possibility to introduce an aldehyde group, arguably one of the most versatile functional groups in target-oriented synthesis, alongside the formation of a new $\mathrm{C}-\mathrm{H}$ bond (Fig. 1A, top $)^{2,23-25}$. Given the importance of creating new $\mathrm{C}-\mathrm{C}$ bonds ${ }^{26}$ and the widespread academic and industrial impact of hydroformylation, the development of carboformylation reactions, wherein a new $\mathrm{C}-\mathrm{C}$ bond is formed instead of a $\mathrm{C}-\mathrm{H}$ bond, would bear enormous synthetic potential as a tool to rapidly prepare densely functionalized aldehydes which are widespread synthetic intermediates (Fig. 1a, bottom).

Despite the promises offered by a carboformylation paradigm, only a single isolated example of carboformylation has been disclosed thus far by Grigg (Fig. 1b) ${ }^{27-29}$. This report clearly captures the challenge of developing such a reaction, as it relies on a carefully designed intramolecular iodoarylalkene substrate undergoing a "molecular queuing" process, involving a hydrosilane and $\mathrm{CO}$, to surgically manipulate the selective formation of three sequential bonds and generate the desired cyclized aldehyde product (Fig. 1b, top). The severity of the method's limitations can be illustrated in an example where shortening the tether length by a single atom led to a different product arising from double insertion of $\mathrm{CO}$ (Fig. 1b, bottom $)^{30}$. This extremely limited reactivity clearly highlights the universal challenges which have plagued the development of intermolecular carboformylation reactions (Fig. 1c): (1) the need for a catalyst system that can delicately mediate a single and selective incorporation of one molecule of $\mathrm{CO}$ in the presence of an excess of $\mathrm{CO}$ gas; (2) the nearly impossible task to orchestrate an intermolecular, chemoselective four-component reaction (an electrophile, $\mathrm{CO}$, a hydride source and an unsaturated substrate), a process which can potentially lead to the formation of more than 10 different products ${ }^{25,30-35}$; (3) the use of toxic and highly flammable CO itself, which has likely limited further research on this topic.

We hypothesized that the challenges of developing an intermolecular carboformylation reaction could potentially be addressed by using acid chlorides as reagents ${ }^{20-22}$. We reasoned that they could simultaneously act as an atom-economic source of a carbon electrophile and $\mathrm{CO}$ in a catalytic carboformylation reaction proceeding through a $\mathrm{CO}$ deinsertion/reinsertion process involving a 
sequence of $\mathrm{C}-\mathrm{C}$ bond cleavage and formation events, mediated by a transition metal catalyst, resulting in a unique combination of well-established elementary steps (Fig. 1d) ${ }^{36,37}$. The use of acid chlorides appeared ideal for several reasons: (1) only 1 equivalent of $\mathrm{CO}$ would be present in the entire reaction system, preventing additional insertions often observed using excess $\mathrm{CO}$ gas as a reagent; (2) acid chlorides can rapidly react with a low valent transition metal to generate the corresponding aryl-M complex (II, e.g. $\mathrm{M}=\mathrm{Pd}$ ), which can then add across a triple bond; (3) the intermediacy of a $\mathrm{M}-\mathrm{Cl}$ bond should significantly slow down any undesirable $\mathrm{C}-\mathrm{Cl}$ reductive elimination prior to the programmed $\mathrm{CO}$ reinsertion ${ }^{38-41}$. These essential features should lead to the formation of the final acyl complex (IV) which can then be irreversibly trapped by a hydride, to develop a general platform for the elusive carboformylation reaction ${ }^{12,13}$. However, a daunting challenge to be addressed in this strategy is to prevent any premature trapping of several closely related organopalladium intermediates (I, II and III) generated under the reaction conditions.

Herein, we report an intermolecular carboformylation reaction of internal alkynes using aroyl chlorides as a dual aryl and $\mathrm{CO}$ source. We describe a programmed $\mathrm{CO}$ deinsertion/reinsertion process in which an aromatic acid chloride is formally deconstructed to aryl, $\mathrm{CO}$ and $\mathrm{Cl}$ subcomponents by a Pd catalyst. The individual subcomponents are then shuffled with an alkyne, and reacted with a silane in a programmed order, providing a conceptual framework for the realization of a carboformylation reaction (Fig. 1d). Using bulky hydrosilane reductants, we were able to transform a broad range of alkynes into the corresponding functionalized, tetrasubstituted $\alpha, \beta$-unsaturated aldehydes. The generality of this unique process is further demonstrated by an extension of this reactivity, to the chemodivergent formation of three other valuable carbonyl derivatives, highlighting the potential of this strategy to unlock novel carbonylation reactions.

\section{Results and discussion}

To test our initial hypothesis, we chose internal alkynes and aromatic acid chlorides as test substrates to prepare highly functionalized $\alpha, \beta$-unsaturated aldehydes which are normally accessed through tedious multi-step synthesis ${ }^{24,42}$. After observing small amounts of the product using a wide variety of different phosphine ligands (see Supplementary Section 2), we reasoned that selectively intercepting the right Pd intermediate (IV) with a hydride source would be crucial to improve the yield of the product. We took advantage of the vast palette of commercially available hydrosilanes exhibiting different steric profiles to probe their influence on the reactivity. A clear correlation between the yield of the desired product relative to the steric bulk of the silane could be observed. Using smaller silanes, a larger amount of the undesired aromatic aldehyde was generated, presumably due to premature reduction of $\mathrm{Pd}$ intermediate I (Fig. 1d), whereas sterically congested silanes, $i \mathrm{Pr}_{3} \mathrm{SiH}$ and $\left(i \mathrm{Pr}_{2} \mathrm{HSi}\right)_{2} \mathrm{O}$, resulted in the highest yields of the desired product. While the desired transformation was observed for a wide variety of phosphine ligands (Supplementary Section 2), after systematic fine-tuning, two phosphine ligands, BISBI (2,2'bis(diphenylphosphinomethyl)-1,1'-biphenyl) and TDMPP (tris(2,6dimethoxyphenyl)phosphine), were found to be optimal, therefore resulting in two complementary conditions for the reaction depending on the steric confinement of the aroyl chloride substrate (1, 
$\mathbf{1 4}, \mathbf{1 8})^{43,44}$. The highly sterically encumbered nature of TDMPP may suppress the coordination of a second phosphine to the metal atom. Instead the weakly coordinating methoxy group may transiently occupy the vacant site and be displaced by the comparatively smaller ligands (i.e. CO or an alkyne), in which their coordination is a prerequisite for the desired transformation. Similarly, a weakly chelating bidentate phosphine ligand, BISBI, which possesses a large bite angle and flexible structure, may act so that one of the $\mathrm{P}$ atoms binds in a hemi-labile manner, thus allowing for the generation of a vacant coordination site. With the optimized catalyst systems, the reaction of an equimolar ratio of all reaction components (an acid chloride/alkyne/silane $=1: 1: 1$ ) gave the desired carboformylation as the major product (1: $44 \%$ yield, 18: $40 \%$ yield, see Supplementary Section 2.6) alongside the side-products (the corresponding arene and aryl aldehyde) formed by premature reduction. To ensure the reaction was driven to high yield versus the alkyne as the limiting reagent, a 2-fold excess of acid chloride and silane was used, resulting in an increased yield (1: 71\% yield, 18: 72\% yield, see Supplementary Section 2.6), albeit, with an increase in side-product formation. For the reaction scope, the equivalents of both acid chloride and silane (1.5 to 3.0 equiv.) versus the alkyne were employed according to the steric congestion of the acid chloride (see Supplementary Section 3).

With the optimized conditions in hand, we set out to investigate the scope of this carboformylation. Various (hetero)aroyl chlorides bearing ortho-substituents successfully participate in this Pdcatalyzed carboformylation with 4-octyne, producing the desired $\alpha, \beta$-unsaturated aldehydes with

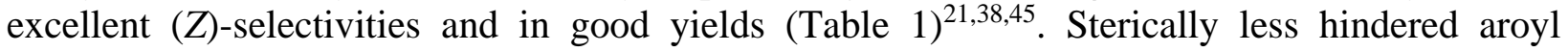
chlorides provided moderate yields accompanied by 5-20\% of the corresponding $(E)$-isomer, suggesting that the ortho-substituents of aroyl chlorides impede $E / Z$ isomerization ${ }^{11,12}$. While sterically encumbered aroyl chlorides can be used in near stoichiometric amounts (1.5 equivalent versus an alkyne) for high yields, a 2- to 3-fold excess of the other acid chlorides were employed to obtain synthetically relevant yields. Methyl-, ethyl-, benzyl- and trifluoromethyl ethers $(\mathbf{3}, \mathbf{5}$, 21, 22), esters $(\mathbf{4}, \mathbf{2 4})$, nitriles $(\mathbf{6}, \mathbf{2 6})$, trifluoromethyl groups $(\mathbf{7}, \mathbf{2 8})$, ketones $(\mathbf{1 0})$ and sulfonamides (29) are tolerated. Aryl halogenides $(\mathrm{Cl}(\mathbf{8}, \mathbf{1 5}), \mathrm{Br}(\mathbf{2 5}))$ and aryl boronate esters (23), which are, respectively, ubiquitous carbon electrophiles and nucleophiles in conventional cross-coupling reactions, are compatible. Furthermore, a benzyl protecting group (22) remained intact under the reducing reaction conditions, namely a Pd catalyst and a hydride source, illustrating the orthogonality and chemoselectivity of this new reaction. Heteroaromatic acid chlorides, including thiophene (11), benzofuran (12), pyridine (13), isoxazole (16) and thiazole (30) derivatives, are also effective reaction partners. In addition, two derivatives of carboxylic acid-containing pharmaceutical compounds (probenecid (29) and febuxostat (30)) participated in this carboformylation. Notably, aroyl chlorides bearing electron-withdrawing substituents showed moderate yields and diminished stereoselectivity. GC analysis of the crude reaction mixtures showed an increased yield of a side product arising from competing hydroarylation, indicating that CO reinsertion (III to IV) is less favored with electron-poor substrates. Finally, using isotopically 40 labelled reagents, the $\mathrm{D}$ and ${ }^{13} \mathrm{C}$ labels were efficiently incorporated into the desired products $(\mathbf{2}$, 20), highlighting the synthetic potential of this method as an addition to medicinal chemistry's toolbox for the preparation of isotopically labelled $\alpha, \beta$-unsaturated aldehydes ${ }^{17,46}$. Gratifyingly, the reaction could be performed on a multi-gram scale $(25 \mathrm{mmol})$, delivering the product (1) in excellent yield $(83 \%, 4.8 \mathrm{~g})$ with complete stereoselectivity. This result clearly demonstrates the 45 potential of this reaction for preparative synthesis without the need for any specialized equipment ${ }^{47}$, such as autoclaves or two-chamber reaction vessels. 
Next, the generality of this method with respect to symmetrical and unsymmetrical internal alkynes was explored using $o-, p$-toluoyl and mesitoyl chlorides as representative acid chloride substrates (Table 2). Alkyl chlorides (34), phthalimides (40), esters (41-44, 47-49), carbamates (45), alkenes (52), amides (53), silyl ethers (57) and aldehydes (62) were all tolerated. Several internal alkynes tethered with a polar functional group such as phthalimides, esters, carbamates and ethers were also probed to explore whether directing effects could influence the reaction's regioselectivity. While most of them resulted in only slightly improved or negligible regioselectivity, an ester at the $\beta$-position relative to the alkyne led to a synthetically useful ratio of separable isomers (up to $3: 1, \mathbf{4 2}-\mathbf{4 4})^{19}$. Additionally, high regioselectivity was obtained when a highly sterically encumbered alkyne is reacted with a bulky aroyl chloride (35), displaying preferential aryl insertion at the distal position relative to the bulky group, presumably to minimize steric repulsion. Next, aryl-alkyl and diaryl alkynes $(\mathbf{5 0 - 5 3}, \mathbf{6 4})$ were shown to be suitable substrates for this transformation, offering new opportunities to access novel conjugated aldehydes with potential applications in imaging and organic materials ${ }^{48-51}$. With regards to the regioselectivity in this class of substrates, electronic effects seem to prevail slightly over steric effects since arylation occurred at the more hindered position of an alkyne, resulting in geminal diaryl functionalities (51). While terminal alkynes failed to deliver the anticipated product, likely because of polyinsertion sidereactions $^{44}$, we found that a number of alkyl-silyl (54-57), aryl-silyl alkynes (58-62), and even an aryl-boryl alkyne (63) reacted with complete regio- and stereoselectivity. While being valuable products in their own right, they also grant access, after deprotection, to synthetically desirable products not directly accessible through the direct carboformylation of terminal alkynes.

We next investigated whether this programmed $\mathrm{CO}$ de/reinsertion process would allow for the inclusion of alternative nucleophiles, other than a hydride, as well as different pathways (i.e. $\beta$-H elimination) depending on the structure of the acid chloride reagent. Such extensions would allow for a chemodivergent and modular process, which is enabled by the discovery of this new strategy. As a proof-of-concept, we were interested in replacing both the hydride with an aryl nucleophile, to generate either an aldehyde or a ketone, as well as the aroyl chloride electrophile with an aliphatic acid chloride, to allow for potential reactivity divergences. Such a set of experiments involving all the permutations would enable us to transform the same alkyne substrate into four kinds of diversified products under a nearly identical set of reaction conditions (Fig. 2a). Remarkably, with only subtle modification of the reaction conditions, four different carbonylated products could indeed be obtained in good yields and excellent stereoselectivities, making this programmed $\mathrm{CO}$ de/reinsertion process a modular tool for the realization of a diverse range of carbonylation reactions (Fig. 2b). Apart from carboformylation, a CO-free carboacylation could also be realized by using stannane reagents as carbon nucleophiles in place of the silane (67-71). In an alternative pathway, aliphatic acid chlorides can undergo $\beta-\mathrm{H}$ elimination to release an alkene. The $\mathrm{H}-\mathrm{Pd}(\mathrm{II})-\mathrm{Cl}$ intermediate and $\mathrm{CO}$ generated from this step can then be combined with 40 a hydride or aryl nucleophile, to unlock the corresponding hydroformylation and hydroacylation reactions $(\mathbf{6 5 - 6 6}, \mathbf{7 2 - 7 5})$, thus significantly expanding the breadth of the strategy. It is noteworthy that unlike the carbofomylation reaction presented above, a terminal alkyne can undergo both carbo- and hydroacylation $(\mathbf{7 1}, \mathbf{7 5})$. The inverse regioselectivity between a silyl substituted alkyne (70) and a terminal alkyne (71) shown in this carboacylation results in yet another handle for synthetic control. 


\section{Conclusion}

In summary, we have developed a reaction for the carboformylation of alkynes. Not only does this reaction add a versatile aldehyde moiety across an alkyne, but it does so concomitantly while forming a new $\mathrm{C}-\mathrm{C}$ bond, offering a facile route to densely functionalized aldehydes which are otherwise challenging to access. The enabling feature of this reaction is the use of an aroyl chloride as both a carbon and $\mathrm{CO}$ source through a programmed $\mathrm{CO}$ de/reinsertion strategy. In a broader context, this reactivity clearly highlights the potential of this approach for the development of a vast array of new, CO-free carbonylation reactions as demonstrated in a striking example of chemodivergent catalysis.

\section{References}

1. Bertleff, W., Roeper, M. \& Sava, X. in Ullmann's Encyclopedia of Industrial Chemistry 7395 (Wiley-VCH, Weinheim, 2007).

2. Börner, A. \& Franke, R. Hydroformylation: Fundamentals, Processes, and Applications in Organic Synthesis (Wiley-VCH, Weinheim, 2016).

3. Cornils, B., Herrmann, W. A. \& Rasch, M. Otto Roelen, pioneer in industrial homogeneous catalysis. Angew. Chem. Int. Ed. 33, 2144-2163 (1994).

4. Kiss, G. Palladium-catalyzed Reppe carbonylation. Chem. Rev. 101, 3435-3456 (2001).

5. Peng, J.-B., Wu, F.-P. \& Wu, X.-F. First-row transition-metal-catalyzed carbonylative transformations of carbon electrophiles. Chem. Rev. 119, 2090-2127 (2019).

6. Bai, Y., Davis, D. C. \& Dai, M. Natural product synthesis via palladium-catalyzed carbonylation. J. Org. Chem. 82, 2319-2328 (2017).

7. Hood, D. M. et al. Highly active cationic cobalt(II) hydroformylation catalysts. Science 367, 542-548 (2020).

8. Yang, J. et al. Direct synthesis of adipic acid esters via palladium-catalyzed carbonylation of 1,3-dienes. Science 366, 1514-1517 (2019).

9. Willcox, D. et al. A general catalytic $\beta-\mathrm{C}-\mathrm{H}$ carbonylation of aliphatic amines to $\beta$-lactams. Science 354, 851-857 (2016).

10. Kinney, R. G. et al. A general approach to intermolecular carbonylation of arene $\mathrm{C}-\mathrm{H}$ bonds to ketones through catalytic aroyl triflate formation. Nat. Chem. 10, 193-199 (2018).

11. Schoenberg, A. \& Heck, R. F. Palladium-catalyzed formylation of aryl, heterocyclic, and vinylic halides. J. Am. Chem. Soc. 96, 7761-7764 (1974). 
12. Baillargeon, V. P. \& Stille, J. K. Palladium-catalyzed formylation of organic halides with carbon monoxide and tin hydride. J. Am. Chem. Soc. 108, 452-461 (1986).

13. Pri-Bar, I. \& Buchman, O. Reductive formylation of aromatic halides under low carbon monoxide pressure catalyzed by transition-metal compounds. J. Org. Chem. 49, 4009-4011 (1984).

14. Wu, X.-F. et al. Development of a General Palladium-Catalyzed Carbonylative Heck Reaction of Aryl Halides. J. Am. Chem. Soc. 132, 14596-14602 (2010).

15. Wu, L., Liu, Q., Jackstell, R. \& Beller, M. Carbonylations of alkenes with CO surrogates. Angew. Chem. Int. Ed. 53, 6310-6320 (2014).

16. Morimoto, T. \& Kakiuchi, K. Evolution of carbonylation catalysis: no need for carbon monoxide. Angew. Chem. Int. Ed. 43, 5580-5588 (2004).

17. Friis, S. D., Lindhardt, A. T. \& Skrydstrup, T. The development and application of twochamber reactors and carbon monoxide precursors for safe carbonylation reactions. Acc. Chem. Res. 49, 594-605 (2016).

18. Murphy, S. K., Park, J.-W., Cruz, F. A. \& Dong, V. M. Rh-catalyzed C-C bond cleavage by transfer hydroformylation. Science 347, 56-60 (2015).

19. Tan, G., Wu, Y., Shi, Y. \& You, J. Syngas-free rhodium-catalyzed highly regioselective transfer hydroformylation of alkynes to $\alpha, \beta$-unsaturated aldehydes. Angew. Chem. Int. Ed. 58, 7440-7444 (2019).

20. Fang, X., Cacherat, B. \& Morandi, B. CO- and HCl-free synthesis of acid chlorides from unsaturated hydrocarbons via shuttle catalysis. Nat. Chem. 9, 1105-1109 (2017).

21. Lee, Y. H. \& Morandi, B. Metathesis-active ligands enable a catalytic functional group metathesis between aroyl chlorides and aryl iodides. Nat. Chem. 10, 1016-1022 (2018).

22. De La Higuera Macias, M. \& Arndtsen, B. A. Functional group transposition: a palladiumcatalyzed metathesis of $\mathrm{Ar}-\mathrm{X} \sigma$-bonds and acid chloride synthesis. J. Am. Chem. Soc. 140, 10140-10144 (2018).

23. Johnson, J. R., Cuny, G. D. \& Buchwald, S. L. Rhodium-catalyzed hydroformylation of internal alkynes to $\alpha, \beta$-unsaturated aldehydes. Angew. Chem. Int. Ed. 34, 1760-1761 (1995).

24. Zhang, S., Neumann, H. \& Beller, M. Synthesis of $\alpha, \beta$-unsaturated carbonyl compounds by carbonylation reactions. Chem. Soc. Rev. 49, 3187-3210 (2020).

25. Matsuda, I., Ogiso, A., Sato, S. \& Izumi, Y. An efficient silylformylation of alkynes catalyzed by $\mathrm{Rh}_{4}(\mathrm{CO})$ 12. J. Am. Chem. Soc. 111, 2332-2333 (1989).

26. Marek, I., Chinkov, N. \& Banon-Tenne, D. in Metal-Catalyzed Cross-Coupling Reactions (eds de Meijere, A. \& Diederich, F.) 395-478 (Wiley-VCH, Weinheim, 2004).

27. Brown, S., Clarkson, S., Grigg, R. \& Sridharan, V. Palladium-catalysed cyclisationcarboformylation. molecular queuing cascades. J. Chem. Soc., Chem. Commun. 1135-1136 (1995).

28. Brown, S. et al. Palladium catalysed queuing processes. part 1: termolecular cyclization-anion capture employing carbon monoxide as a relay switch and hydride, organotin(IV) or boron reagents. Tetrahedron 57, 1347-1359 (2001). 
29. Negishi, E., Copéret, C. in Handbook of Organopalladium Chemistry for Organic Synthesis (ed Negishi, E.) 1431-1448 (Wiley, New York, 2002).

30. Van den Hoven, B. G. \& Alper, H. Innovative synthesis of 4-carbaldehydepyrrolin-2-ones by zwitterionic rhodium catalyzed chemo- and regioselective tandem cyclohydrocarbonylation/CO insertion of $\alpha$-Imino alkynes. J. Am. Chem. Soc. 123, 1021410220 (2001).

31. Fuji, K., Morimoto, T., Tsutsumi, K. \& Kakiuchi, K. Rh(I)-catalyzed CO gas-free cyclohydrocarbonylation of alkynes with formaldehyde to $\alpha, \beta$-butenolides. Chem. Commun. 3295-3297 (2005).

32. Peng, J.-B., Wu, F.-P., Spannenberg, A. \& Wu, X.-F. Palladium-catalyzed tunable carbonylative synthesis of enones and benzofulvenes. Chem. Eur. J. 25, 8696-8700 (2019).

33. Fujihara, T., Tatsumi, K., Terao, J. \& Tsuji, Y. Palladium-catalyzed formal hydroacylation of allenes employing acid chlorides and hydrosilanes. Org. Lett. 15, 2286-2289 (2013).

34. Kokubo, K., Matsumasa, K., Miura, M. \& Nomura, M. Rhodium-catalyzed reaction of aroyl chlorides with alkynes. J. Org. Chem. 61, 6941-6946 (1996).

35. Yasukawa, T., Satoh, T., Miura, M. \& Nomura, M. Iridium-catalyzed reaction of aroyl chlorides with internal alkynes to produce substituted naphthalenes and anthracenes. J. Am. Chem. Soc. 124, 12680-12681 (2002).

36. Wang, P., Rao, H., Zhou, F., Hua, R. \& Li, C.-J. Ruthenium-catalyzed aldehyde functionality reshuffle: selective synthesis of $E$-2-arylcinnamaldehydes from $E$ - $\beta$-bromostyrenes and aryl aldehydes. J. Am. Chem. Soc. 134, 16468-16471 (2012).

37. Chen, P., Billett, B. A., Tsukamoto, T. \& Dong, G. "Cut and Sew" Transformations via transition-metal-catalyzed carbon-carbon bond activation. ACS Catal. 7, 1340-1360 (2017).

38. Tsuji, J. in Handbook of Organopalladium Chemistry for Organic Synthesis (ed Negishi, E.) 2643-2653 (Wiley, New York, 2002).

39. Shen, X., Hyde, A. M. \& Buchwald, S. L. Palladium-catalyzed conversion of aryl and vinyl triflates to bromides and chlorides. J. Am. Chem. Soc. 132, 14076-14078 (2010).

40. Petrone, D. A., Ye, J. \& Lautens, M. Modern transition-metal-catalyzed carbon-halogen bond formation. Chem. Rev. 116, 8003-8104 (2016).

41. Malapit, C. A., Ichiishi, N. \& Sanford, M. S. Pd-catalyzed decarbonylative cross-couplings of aroyl chlorides, Org. Lett. 19, 4142-4145 (2017).

42. Flynn, A. B. \& Ogilvie, W. W. Stereocontrolled synthesis of tetrasubstituted olefins. Chem. Rev. 107, 4698-4745 (2007).

43. Casey, C. P. et al. Diphosphines with natural bite angles near $120^{\circ}$ increase selectivity for $n$ aldehyde formation in rhodium-catalyzed hydroformylation. J. Am. Chem. Soc. 114, 55355543 (1992).

44. Trost, B. M., Sorum, M. T., Chan, C., Harms, A. E. \& Rühter, G. Palladium-catalyzed additions of terminal alkynes to acceptor alkynes. J. Am. Chem. Soc. 119, 698-708 (1997).

45. El-Khawaga, A. M., Roberts, R. M. \& Sweeney, K. M. Transacylations between sterically 
46. Gauthier, Jr., D. R., Rivera, N. R., Yang, H., Schultz, D. M. \& Shultz, C. S. Palladiumcatalyzed carbon isotope exchange on aliphatic and benzoic acid chlorides. J. Am. Chem. Soc. 140, 15596-15600 (2018).

47. Natta, G., Ercoli, R., Castellano, S. \& Barbieri, F. H. The influence of hydrogen and carbon monoxide partial pressures on the rate of the hydroformylation reaction. J. Am. Chem. Soc. 76, 4049-4050 (1954).

48. Cnossen, A., Browne, W. R. \& Feringa, B. L. in Molecular Machines and Motors (eds Credi, A., Silvi, S. \& Venturi, M.) 139-162 (Springer, Berlin, 2014).

49. Meier, H. The photochemistry of stilbenoid compounds and their role in materials technology. Angew. Chem. Int. Ed. 31, 1399-1420 (1992).

50. Mei, J., Leung, N. L. C., Kwok, R. T. K., Lam, J. W. Y. \& Tang, B. Z. Aggregation-induced emission: together we shine, united we soar! Chem. Rev. 115, 11718-11940 (2015).

51. Chan, J., Dodani, S. C. \& Chang, C. J. Reaction-based small-molecule fluorescent probes for chemoselective bioimaging. Nat. Chem. 4, 973-984 (2012).

\section{Data availability}

Crystallographic data for the structures reported in this Article have been deposited at the Cambridge Crystallographic Data Centre, under deposition numbers CCDC 1998285 (10), 2018417 (33), 2000217 (36, minor), 2000218 (53), 2018418 (59), 2018419 (59-Z), 1998290 (62), 1998292 (64), 1998293 (L08) and 1998299 (76). Copies of the data can be obtained free of charge via https://www.ccdc.cam.ac.uk/structures/. All other data is available in the main text or the Supplementary Information.

\section{Acknowledgements}

We acknowledge the ETH Zürich, the European Research Council under the European Union's Horizon 2020 research and innovation program (Shuttle Cat, Project ID: 757608), and LG Chem (fellowship to Y.H.L.) for financial support. We thank the NMR, MS (MoBiAS) and X-ray (SMoCC) service departments at ETH Zürich for technical assistance.

\section{Author contributions}

Y.H.L. designed and discovered the reaction. Y.H.L. and E.H.D. performed the scope of the reaction. B.M. supervised the research. All authors contributed to manuscript writing and editing.

\section{Competing interests}

The authors declare no competing interests. 


\section{Figure legends}

a

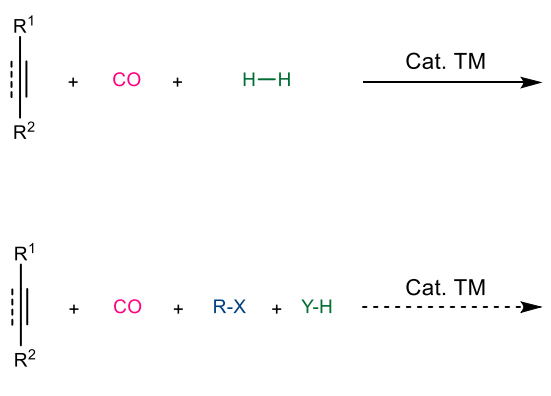

c

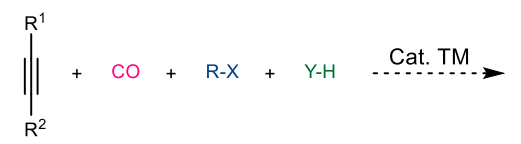

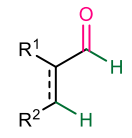

hydroformylation (mono-functionalization)<smiles>[R]C([R])=C([R])C=O</smiles>

carboformylation (di-functionalization) b

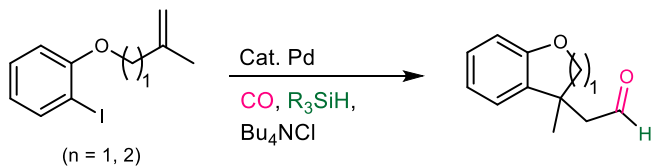<smiles>C=C(C)Cc1ccccc1I</smiles>

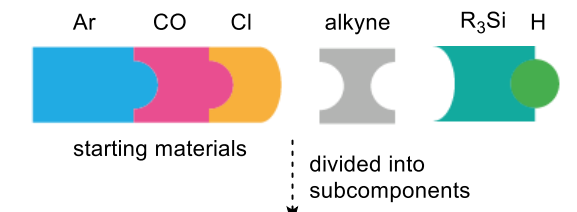

desired product<smiles>[R]C(=O)/C([Y])=C(\[R])C([R])=O</smiles><smiles>[R]C([R])=C([R])[18OH]</smiles>

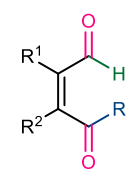

$\underbrace{C}_{O}$

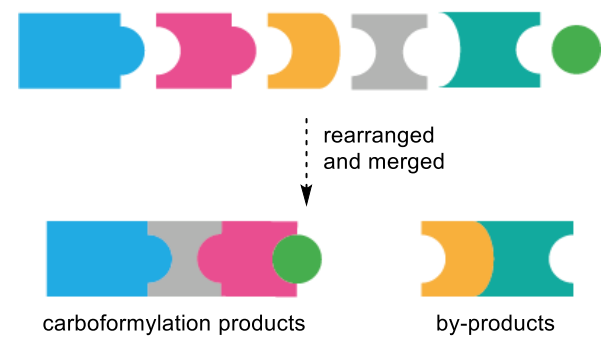

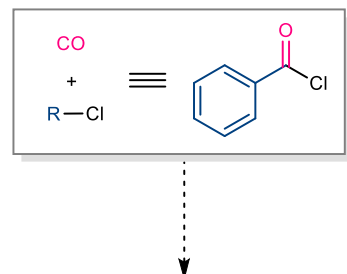

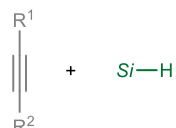

Cat. TM
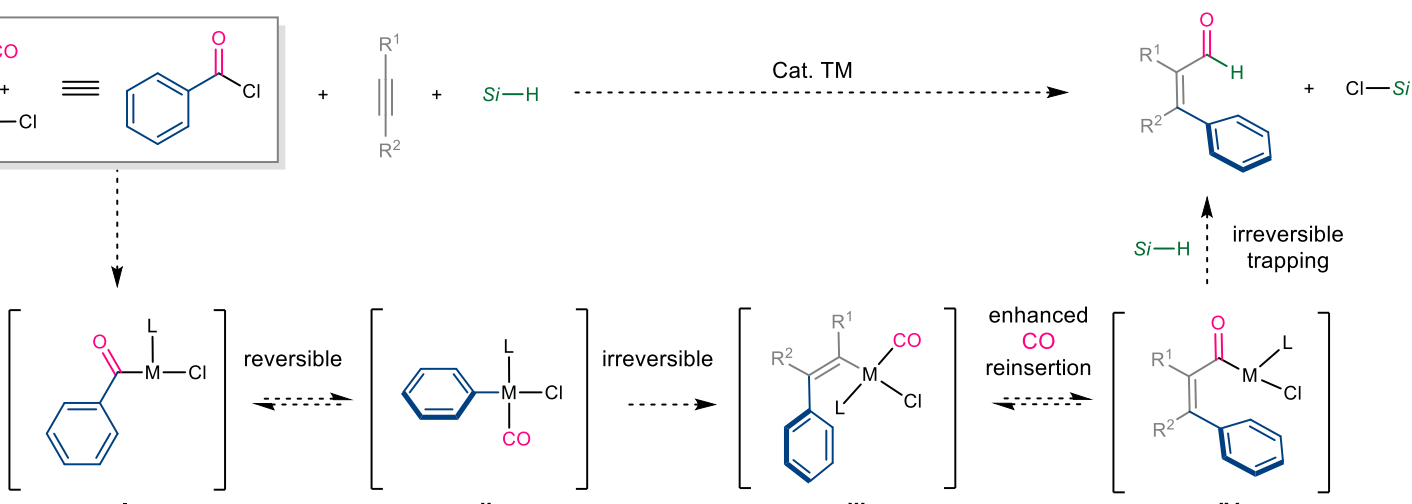

Fig. 1. Comparison between conventional hydroformylation and carboformylation, and the conceptual blueprint for a general intermolecular carboformylation. a, Hydroformylation is a reaction which is performed on a 10-million-ton scale per year; is the subject of $>10,000$ publications and patents to date; and is widely used in industry and academia. By contrast, the analogous carboformylation reaction, which has considerable synthetic potential, has a single reported example which is intramolecular and is the topic of only 2 publications (Grigg, ref. 26- 
27). b, The intramolecular carboformylation developed by Grigg (1995), which utilizes a carefully engineered substrate, and changes in tether length results in a different product. $\mathbf{c}$, Challenges for the intermolecular carboformylation using a conventional approach include the possible formation of products (>10); non-selective insertion of $\mathrm{CO}$, where excess $\mathrm{CO}$ favors polycarbonylation. The use of pressurized $\mathrm{CO}$ gas also limits R\&D efforts in academia. d, Our conceptual blueprint - the deconstruction of acid chlorides, as an aryl and $\mathrm{CO}$ source, and its merging across an alkyne substrate in programmed order. e, Our strategy - the oxidative addition of a metal catalyst into the acyl-Cl bond of an acid chloride (I); reversible $\mathrm{CO}$ deinsertion (II); a migratory insertion reaction with an unsaturated $\mathrm{C}-\mathrm{C}$ bond to generate the alkenyl-M complex (III); reversible $\mathrm{CO}$ reinsertion (IV); The carboformylated product resulted from irreversible trapping by a hydrosilane. Cat. TM, transition metal catalyst. R-X, carbon electrophile. Y-H, hydride source. Cat. Pd, palladium catalyst. $\mathrm{Si}-\mathrm{H}$, hydrosilane. $\mathrm{Cl}-\mathrm{Si}$, chlorosilane. L, ligand. 

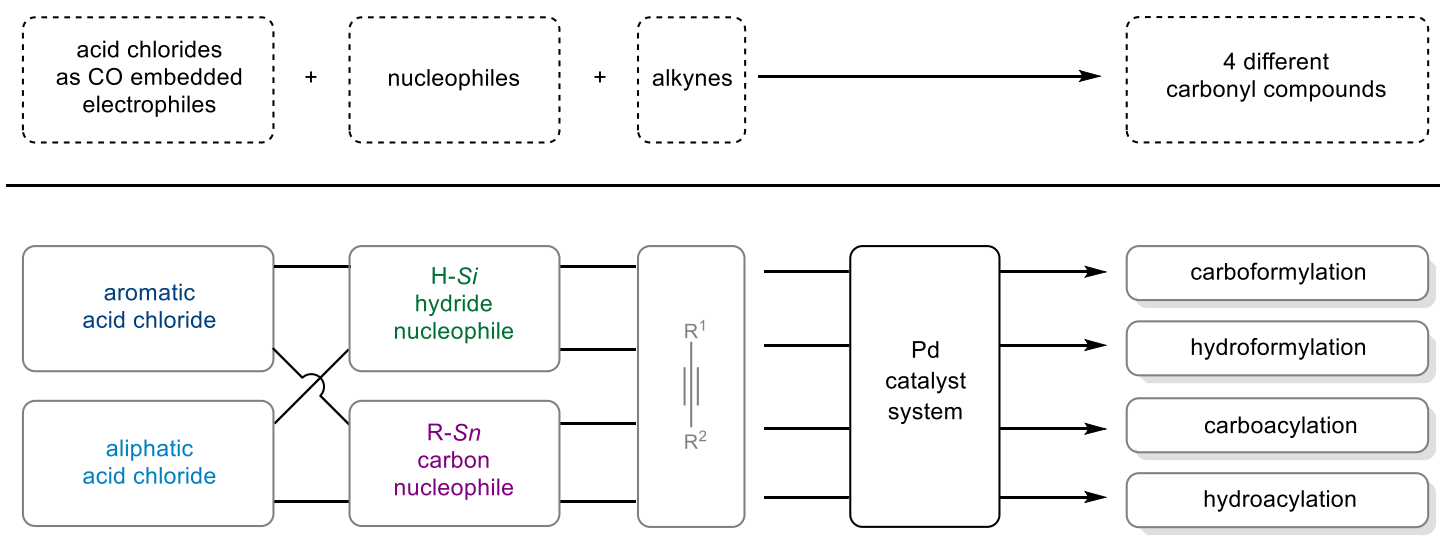

b

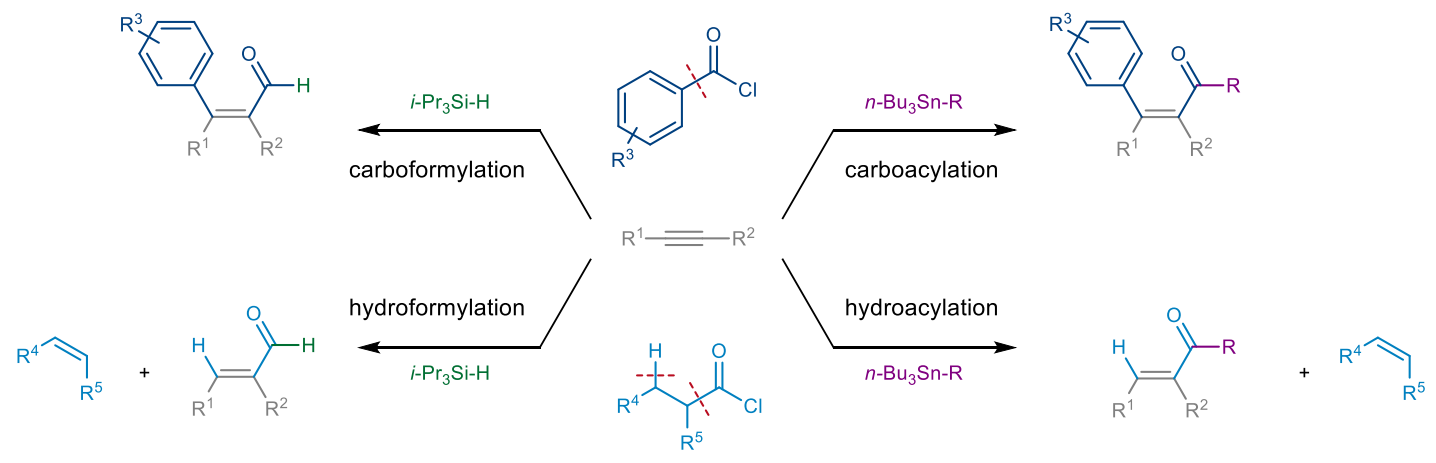

c, scope for carboacylation
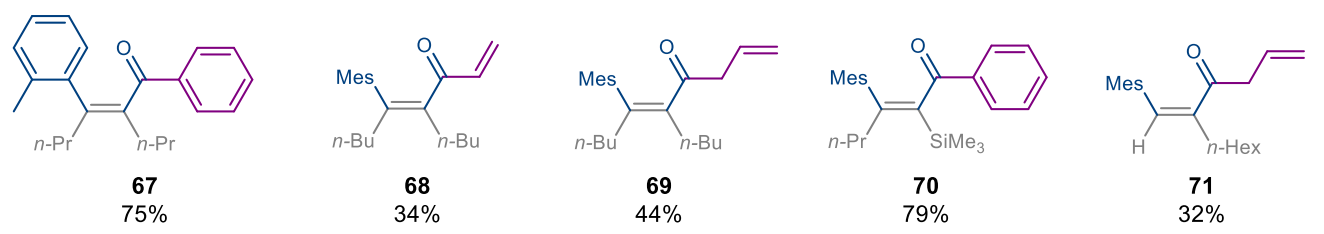

e, scope for hydroacylation
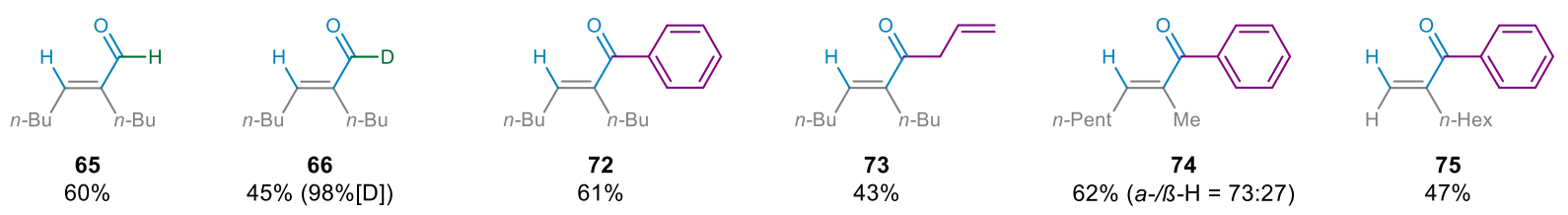

Fig. 2. Chemodivergent carbonylation reaction. a, The modular process is capable of producing a diverse range of carbonyl-containing compounds. b, Chemodivergent carbonylation reactions using different acid chlorides or nucleohpiles can generate (1) carboformylation products; (2) hydroformylation products; (3) carboacylation products; (4) hydroacylation products. c, scope of the carboacylation reaction. d, scope of the hydroformylation reaction. e, scope of the hydroacylation reaction. All yields are isolated as a single stereo- and regioisomers unless stated 
otherwise. For details, see Supplementary Section 4, 5 and 6. H-Si, hydrosilane. R-Sn, aryl, vinyl or allyl stannane. $n$-Hex, $n$-Hexyl. 


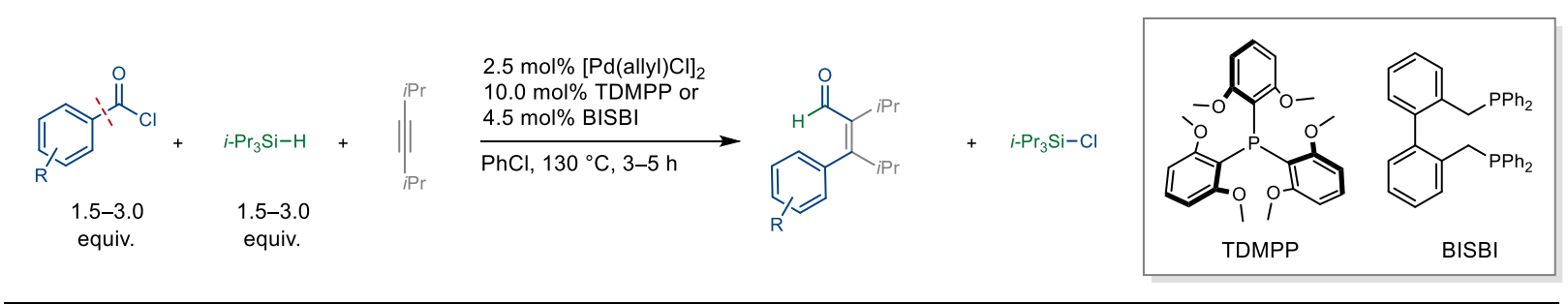

mono-ortho-substituted aroyl chlorides (1 to 13)

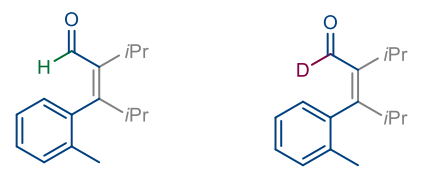

1: $80 \%^{\mathrm{a}}, 65 \%^{\mathrm{b}}$ $82 \%{ }^{a, c}$

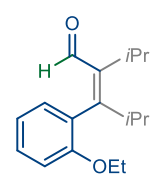

3: $53 \%^{a}$ $58 \%$ b

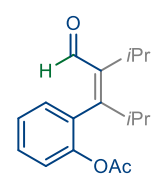

4: $40 \%^{\mathrm{a}}$ $63 \%{ }^{b}$<smiles>CC(C(=O)O)c1ccccc1C(=O)O</smiles>

5: $76 \%^{\mathrm{a}}$ $67 \%$

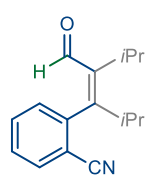

6: $51 \%^{\mathrm{a}}$

$(Z / E=83: 17)$

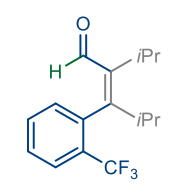

7: $63 \%^{\mathrm{a}}$ $79 \%$ b $83 \%^{a, c, d}(4.8 \mathrm{~g}$, gram scale)<smiles>CC(C)/C(C=O)=C(\c1cccc2ccccc12)C(C)C</smiles>

$70 \%^{\mathrm{b}}$

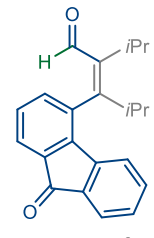

10: $52 \%^{a}$

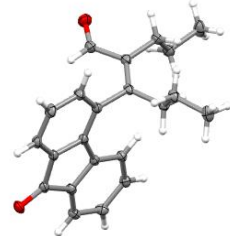

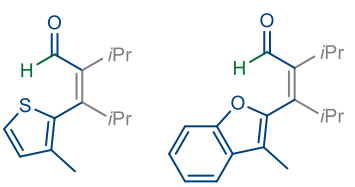

12: $67 \%^{\mathrm{a}}$<smiles>Cc1nc(C(F)(F)F)ccc1/C(C=O)=C\C(C)C</smiles>

13: $66 \%{ }^{a}$

di-ortho-substituted aroyl chlorides (14 to 16 )<smiles>CC(=C(C=O)C(C)C)C(C)C</smiles><smiles>[R]C(C)=C(C=O)C(C)C</smiles><smiles>Cc1onc(-c2ccccc2)c1C(C(C)C)C(C)C</smiles>

14: $82 \%^{\mathrm{a}}$ $97 \%$

16: $42 \%^{a}$

15: $71 \%^{\mathrm{a}}$

non-ortho-substituted aroyl chlorides (17 to 28 )<smiles>COc1ccc(C(C=O)C(C)C)cc1</smiles>

21: $48 \%^{a}$<smiles>CC(C)Oc1ccc(/C(=C(/C=O)C(C)C)C(C)C)cc1</smiles>

22: $44 \%^{a}$

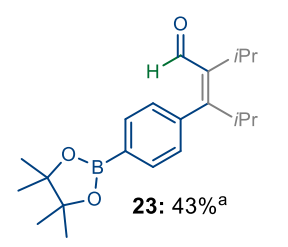

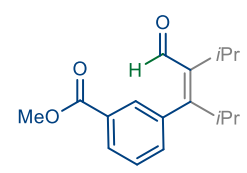

24: $51 \%^{a}$

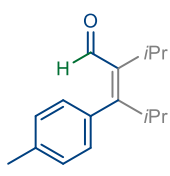

18: $76 \%$ a,e

$(Z / E=88: 12)$ $39 \%$ b,e $(Z / E=94: 6)$

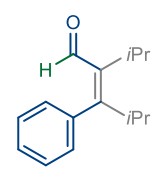

19: $66 \%{ }^{\mathrm{a}, \mathrm{e}}$

$(Z / E=95: 5)$

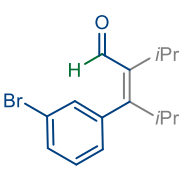

25: $69 \%^{\mathrm{a}, \mathrm{e}}$ $(Z / E=79: 21)$

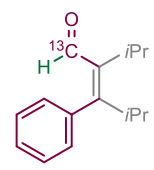

20: $67 \%{ }^{\mathrm{a}, \mathrm{e}}$

(Z/E $=95: 5$, $\left.>99 \%\left[{ }^{13} \mathrm{C}\right]\right)$<smiles>CC(C)/C(C=O)=C(\c1ccc(F)cc1)C(C)C</smiles>

27: $70 \%{ }^{a, e}$

$(Z / E=87: 13)$<smiles>CC(C)/C(C=O)=C(\c1cc(C(F)(F)F)cc(C(F)(F)F)c1)C(C)C</smiles>

28: $57 \%^{a}$

$(Z / E=79: 21)$

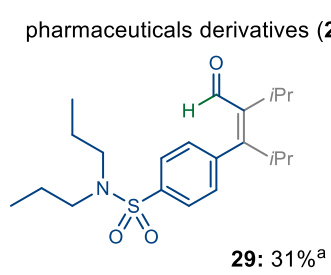

Probenecid derivative

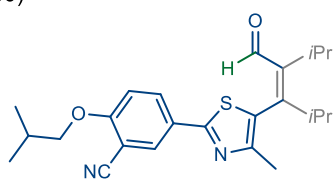

30: $66 \%^{\mathrm{a}}$

Febuxostat derivative

Table 1. Scope with respect to aroyl chlorides. All isolated yields as a single stereoisomer unless otherwise stated. ${ }^{\text {aTDMPP. }}{ }^{\mathrm{b}}$ BISBI. ${ }^{\mathrm{c}}$ Open system. ${ }^{\mathrm{d}} 25 \mathrm{mmol}$ scale. ${ }^{\mathrm{e}}$ Isolated as an inseparable mixture of stereoisomers. $i \mathrm{Pr}$, isopropyl. Mes, Mesityl. 2,6-diClPh, 2,6-Dichlorophenyl. 2,6diFPh, 2,6-Difluorophenyl. Bn, Benzyl. For details, see Supplementary Section 3 and 11. 

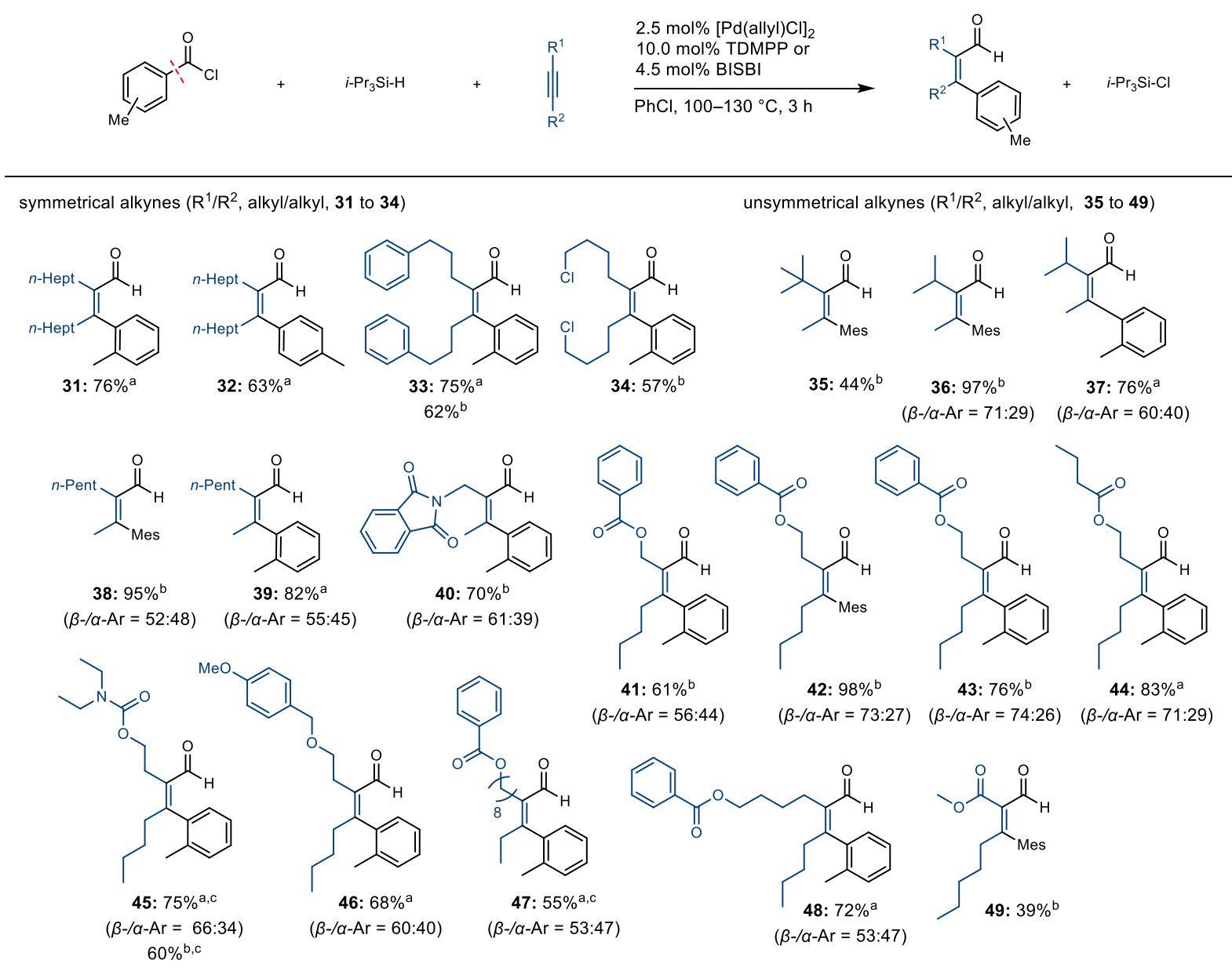

unsymmetrical alkynes $\left(\mathrm{R}^{1} / \mathrm{R}^{2}\right.$, aryl/alkyl, $\mathbf{5 0}$ to $\left.\mathbf{5 3}\right)$

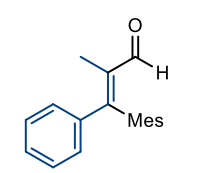

50: $78 \%{ }^{\mathrm{b}}$

$(\alpha-/ \beta-A r=50: 50)$<smiles>CCCC(C=O)=C(C)c1ccccc1</smiles>

51: $72 \%{ }^{\mathrm{b}}$

$(\alpha-/ \beta-\mathrm{Ar}=63: 37)$<smiles>C=CC/C(C=O)=C(/c1ccccc1)[N+](=O)[O-]</smiles>

52: $42 \%^{\mathrm{b}}$

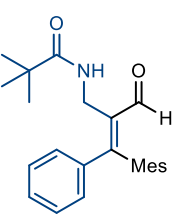

53: $24 \%^{\mathrm{b}}$

unsymmetrical alkynes $\left(\mathrm{R}^{1 / R^{2}}\right.$, aryl/silyl or boryl, 58 to 63$)$<smiles>CC(=C(C=O)C(=O)N(C)C)c1ccc(C)cc1</smiles>

58: $\mathrm{R}=\mathrm{H} ; 70 \%{ }^{\mathrm{b}}$

59: $\mathrm{R}=\mathrm{OMe} ; 43 \%^{\mathrm{b}}, 91 \%^{\mathrm{b}, \mathrm{d}} \quad 62: 65 \%^{\mathrm{b}}$

60: $\mathrm{R}=\mathrm{CN}, 74 \%^{\mathrm{b}}$

61: $\mathrm{R}=\mathrm{CF}_{3} ; 79 \%{ }^{\mathrm{b}}$

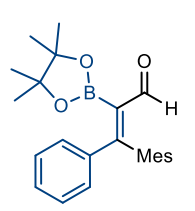

63: $41 \%^{\mathrm{b}}$

64: $38 \%^{\mathrm{b}}$

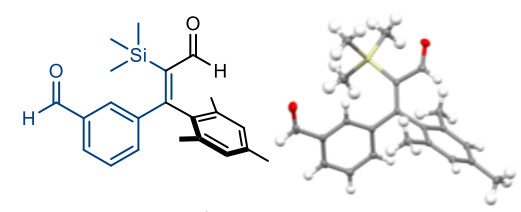

symmetrical alkynes $\left(R^{1} / R^{2}\right.$, aryl/aryl, 64$)$

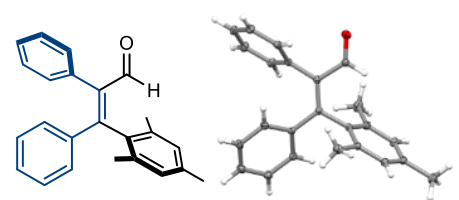

54: $78 \%^{\mathrm{b}}$

55: $97 \%$

56: $56 \%$

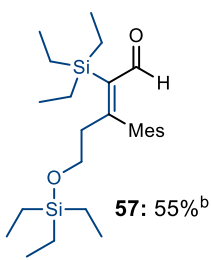

Table 2. Scope with respect to internal alkynes. All yields are isolated as a single stereo- and regioisomer unless otherwise stated. ${ }^{\text {aTDMPP. }}{ }^{\text {bBISBI. }}$ 'Isolated as an inseparable mixture of regioisomers. ${ }^{\mathrm{d}}$ NMR yield. $n$-Hept, $n$-Heptyl. $n$-Pent, $n$-Pentyl. Mes, Mesityl. For details, see Supplementary Section 3. 


\section{Methods}

As general procedure for mono-ortho-substituted aroyl chloride, in a glovebox, a $4 \mathrm{~mL}$ screw-cap vial equipped with a stirring bar was charged with $[\mathrm{Pd}(\text { allyl }) \mathrm{Cl}]_{2}(2.5 \mathrm{~mol} \%, 6.25 \mu \mathrm{mol}, 2.29 \mathrm{mg}$ or $1.0 \mathrm{~mL}$ as $6.25 \mathrm{mM}$ stock solution in anhydrous chlorobenzene) and TDMPP (10.0 mol\%, 0.025 $\mathrm{mmol}, 11.06 \mathrm{mg}$, or $221.0 \mathrm{mg}$ as $5.0 \mathrm{wt} \%$ stock solution in anhydrous chlorobenzene) then anhydrous chlorobenzene ( $2 \mathrm{~mL}$, or $1 \mathrm{~mL}$ when stock solutions were used). The solution was stirred at room temperature for $10 \mathrm{~min}$. To a pre-mixed solution were added aroyl chloride ( 3 equiv, 0.75 mmol), alkyne ( 1.0 equiv, $0.25 \mathrm{mmol}$ ) and $i \operatorname{Pr} 3 \mathrm{SiH}$ ( 3 equiv, $0.75 \mathrm{mmol}, 118.77 \mathrm{mg}$ ). The reaction mixture was stirred at $130{ }^{\circ} \mathrm{C}$ for $3 \mathrm{~h}$. The reaction was cooled to room temperature then quenched with sodium methoxide ( 3 equiv, $0.75 \mathrm{mmol}, 140 \mu \mathrm{L}$ of $5.4 \mathrm{M}$ solution in methanol, note: the quenching step could be omitted for substrates bearing a base-labile functional group) and diluted with dichloromethane $(2 \mathrm{~mL})$. The resulting solution was filtered through a celite/silica plug and concentrated under reduced pressure. The crude product was purified by column chromatography on silica gel (pentane/dichloromethane, pentane/ethyl acetate or pentane/methyl tert-butyl ether).

Correspondence and requests for materials should be addressed to B.M. 